\title{
Article
}

\section{Brain Imaging Signs and Health-Related Quality of Life after Acute Ischemic Stroke: Analysis of ENCHANTED Alteplase Dose Arm.}

Chen, Xiaoying, Delcourt, Candice, Sun, Lingli, Zhou, Zien, Yoshimura, Sohei, You, Shoujiang, Malavera, Alejandra, ToriiYoshimura, Takako, Carcel, Cheryl, Arima, Hisatomi, Hackett, Maree, Robinson, Thompson, Song, Lili, Wang, Xia, Lindley, Richard I, Chalmers, John and Anderson, Craig S

Available at http://clok.uclan.ac.uk/34308/

Chen, Xiaoying, Delcourt, Candice, Sun, Lingli, Zhou, Zien, Yoshimura, Sohei, You, Shoujiang, Malavera, Alejandra, Torii-Yoshimura, Takako, Carcel, Cheryl et al (2020) Brain Imaging Signs and Health-Related Quality of Life after Acute Ischemic Stroke: Analysis of ENCHANTED Alteplase Dose Arm.

Cerebrovascular Diseases . pp. 1-10. ISSN 1015-9770

It is advisable to refer to the publisher's version if you intend to cite from the work. http://dx.doi.org/10.1159/000509226

For more information about UCLan's research in this area go to

http://www.uclan.ac.uk/researchgroups/ and search for <name of research Group>.

For information about Research generally at UCLan please go to http://www.uclan.ac.uk/research/

All outputs in CLoK are protected by Intellectual Property Rights law, including Copyright law. Copyright, IPR and Moral Rights for the works on this site are retained by the individual authors and/or other copyright owners. Terms and conditions for use of this material are defined in the policies page. 


\section{Brain imaging signs and health-related quality of life after acute ischemic stroke: analysis of ENCHANTED alteplase-dose arm}

Xiaoying Chen BPharm BMgt, ${ }^{1,2}$ Candice Delcourt MD PhD, ${ }^{1,2,3}$ Lingli Sun PhD, ${ }^{4}$ Zien Zhou, $\mathrm{MD}^{1,5}$, Sohei Yoshimura, $\mathrm{MD}, \mathrm{PhD}^{1,6}$, Shoujiang You, $\mathrm{MD}^{7}$, Alejandra Malavera, $\mathrm{MD}^{1}$, Takako Torii-Yoshimura, $\mathrm{MD}^{1,8,9}$, Cheryl Carcel, $\mathrm{MD} \mathrm{PhD}^{1,2,3}$, Hisatomi Arima MD $\mathrm{PhD},{ }^{10}$ Maree L Hackett PhD, ${ }^{1,2,11}$ Thompson Robinson MD, ${ }^{12,13}$ Lily Song PhD,${ }^{1,2,4}$ Xia Wang, PhD,${ }^{1}$ Richard I Lindley MD,${ }^{14}$ John Chalmers MD PhD,${ }^{1}$ Craig S. Anderson MD $\mathrm{PhD},{ }^{1,2,3,4,15}$ for the ENCHANTED Investigators

${ }^{1}$ The George Institute for Global Health, University of New South Wales, NSW, Australia ${ }^{2}$ Faculty of Medicine and Health, University of Sydney, NSW, Australia ${ }^{3}$ Neurology Department, Royal Prince Alfred Hospital, Sydney, NSW, Australia ${ }^{4}$ The George Institute China at Peking University Health Science Centre, Beijing, PR China

${ }^{5}$ Department of Radiology, Ren Ji Hospital, School of Medicine, Shanghai Jiao Tong University, Shanghai, China.

${ }^{6}$ Department of Cerebrovascular Medicine, National Cerebral and Cardiovascular Center, Osaka, Japan.

${ }^{7}$ Department of Neurology, the Second Affiliated Hospital of Soochow University, Suzhou, China.

${ }^{8}$ Division of Neurology, Department of Stroke and Cerebrovascular Diseases, National Cerebral and Cardiovascular Center, Osaka, Japan.

${ }^{9}$ Department of Neurology and Neuroscience, Nagoya City University Graduate School of Medical Science, Nagoya, Japan.

${ }^{10}$ Department of Preventive Medicine and Public Health, Fukuoka University, Fukuoka, Japan

${ }^{11}$ Faculty of Health and Wellbeing, University of Central Lancashire, UK

${ }^{12}$ Department of Cardiovascular Sciences, University of Leicester, Leicester, UK

${ }^{13}$ NIHR Biomedical Research Centre, Leicester, UK

${ }^{14}$ Westmead Clinical School, University of Sydney, NSW, Australia

${ }^{15}$ Heart Health Research Center, Beijing, China

\section{Corresponding Author}

Professor Craig S Anderson

The George Institute for Global Health, PO Box M201, Missenden Rd., NSW 2050, Australia T: +61-2-9993-4500; F: +61-2-9993-4502; E: canderson@georgeinstitute.org.au

Word Count: Main Text: 2728 Abstract: 245 
Short title: Imaging predictors of quality of life in acute ischemic stroke

Keywords: thrombolysis, acute ischemic stroke, quality of life, brain imaging

Subject Terms: quality and outcomes, ischemic stroke

Tables: 3

Figures: 1 


\begin{abstract}
Background and Purpose: The influence of specific brain lesions on health-related quality of life (HRQoL) after acute ischemic stroke (AIS) is uncertain. We aimed to identify imaging predictors of poor HRQoL in alteplase-treated participants of the alteplase-dose arm of the Enhanced Control of Hypertension and Thrombolysis Stroke study (ENCHANTED).
\end{abstract}

Methods: ENCHANTED was an international trial of low- versus standard-dose intravenous alteplase in AIS patients, with functional outcome (modified Rankin scale [mRS]) and HRQoL on the 5-dimension European quality of life scale (EQ-5D) assessed at 90 days postrandomization. Brain images were analysed centrally by trained assessors. Multivariable logistic regression was undertaken in the study population randomly divided (2:1) into training (development) and validation (performance) groups; with age (per 10-year increase), ethnicity, baseline National Institutes of Health stroke scale (NIHSS) score, diabetes mellitus, pre-morbid function (mRS score 0 or 1 ) and proxy respondent, forced into all models. Data are presented with odds ratios (OR) and 95\% confidence intervals (CI).

Results: Eight prediction models were developed and validated in 2526 AIS patients (median age 67.5 years; $38.4 \%$ female; $61.7 \%$ Asian) with complete brain imaging and 90day EQ-5D utility score data. The best performance model included acute ischemic changes in the right (OR 1.69, 95\%CI 1.24-2.29) and deep (OR 1.50, 95\%CI 1.03-2.19) middle cerebral artery regions.

Conclusions: Right-sided and deep ischemia predicts poor HRQoL after AIS. Further research is required to understand mechanisms and appropriate rehabilitation strategies to optimise outcomes after thrombolysed AIS.

Clinical Trial Registration-http://www.clinicaltrials.gov. Unique identifier: NCT01422616 


\section{Introduction}

Acute ischemic stroke (AIS) reduces health-related quality of life (HRQoL) in patients and families from disruption of activities and roles in relation to disability, cognitive dysfunction, and mood disorders[1]. Many predictors of poor post-stroke HRQoL have been identified that include: older age[2-6], female sex[2, 5], non-white ethnicity[3, 5, 7], single marital status [6, 8], low education[9], poor socio-economic status[5], diabetes mellitus[3, 10], greater neurological damage severity[3, 4], depression and/or anxiety[6, 8, 11-15], physical[16] and cognitive[17] impairment[9], disability[11], incontinence[3], proxy-respondents[2, 18-20], poor residential status[21, 22], lack of social support[8, 9, 23], poor social participation[14, $22,24,25]$, and inadequate coping strategies[26-28]. In addition, several brain imaging signs - infarct volume[29, 30], subcortical damage[31, 32], microbleeds[33], white matter change[34], and small vessel disease[35] - have been shown to be associated with poor HRQoL, but only a few as independent predictors generated by developing and validating prediction models, possibly due to low statistical power from small studies. Our aim was to identify brain imaging predictors of poor HRQoL among AIS patients who were treated with alteplase and participated in the alteplase-dose arm of the international Enhanced Control of Hypertension and Thrombolysis Stroke Study (ENCHANTED). This large multi-ethnic clinical cohort that underwent standardized measures and central adjudication of brain images afforded us the opportunity to develop and validate various multivariable models.

\section{Methods}

\section{Data sharing}

Individual patient data used in these analyses can be shared by request from any qualified investigators via the Research Office of The George Institute for Global Health, Australia.

\section{Study design}


ENCHANTED was a multicentre, international, $2 \times 2$ quasi-factorial, prospective, randomized, open, blinded outcome trial that investigated the effects of low- vs. standard-dose intravenous alteplase, and intensive vs. guideline-recommended blood pressure (BP) lowering, in thrombolysis-eligible AIS patients, the details of which are outlined elsewhere[36-40]. This paper pertains to patients of the alteplase-dose arm, which was completed earlier and had all brain imaging analyses finalised before those in the BP lowering arm of the trial. The alteplase-dose arm included 3310 AIS patients randomly assigned to low-dose $(0.6 \mathrm{mg} / \mathrm{kg}$; $15 \%$ as bolus, $85 \%$ as infusion over 1 hour $)$ or standard-dose $(0.9 \mathrm{mg} / \mathrm{kg} ; 10 \%$ as bolus, $90 \%$ as infusion over 1 hour) intravenous alteplase. The study protocol was approved by the appropriate ethics committee at each participating centre, and written informed consent was obtained from each patient or an appropriate surrogate. The study is registered with Clinicaltrials.gov (number NCT01422616).

\section{Outcomes}

Demographic and clinical characteristics, including estimated pre-morbid function (modified Rankin scale [mRS] scores 0 or 1 ; patients with scores $>1$ were excluded) were recorded at the time of enrolment (baseline). Neurological severity was assessed using the National Institutes of Health stroke scale (NIHSS) at baseline, 24 hours, and Day 3 (or earlier on discharge from hospital). The primary outcome for the main trial was functional status on the mRS, assessed by telephone or in-person, by trained independent researchers at 90 days. The primary outcome of these analyzes was the 3 level version of the 5-dimension European quality of life scale (EQ5D) which defines general health states across five dimensions (mobility, self-care, usual activities, pain/discomfort, and anxiety/depression) according to three levels of severity (1=no problems, $2=$ some/moderate problems, and $3=$ severe problems)[41]. Ratings on each subscale of the EQ-5D are synthesized into a single utility score using UK population-based preference weights[42, 43]. Utility scores vary from -0.594 to $1 ; 1$ representing perfect health, 0 death, 
and negative scores health states considered worse than death[43]. For these analyses, 'poor HRQoL' was defined as scores of 2 or 3, defining problems within each dimension of EQ-5D or an overall health utility score $\leq 0.7$ (mean).

Uncompressed images of brain computed tomography (CT), magnetic resonance imaging (MRI) and angiography at baseline and at 24-36 hours follow-up were uploaded into the study brain imaging database in Digital Imaging and Communications in Medicine (DICOM) format identified only by patient's unique study number. Where multiple baseline scans were available for a patient, our analysis preference for assessments was the following: (1) noncontrast CT over plain CT images from CT angiogram or perfusion studies; (2) CT scans with thick slices over those with thin slices; (3) scanning time closest to time of randomization; and (4) MRI when this was the only imaging conducted at baseline. Acute/old ischemic changes and location were confirmed and/or justified based on follow-up scans where available. Analysis of the non-hemorrhagic component of the images was conducted by a research team with a background in neurology (1 neuroradiologist, 8 stroke neurologists, and 2 stroke neurology trainees) and standardized training via the ACCESS training module (www.ed.ac.uk/edinburgh-imaging/access). More details about the imaging analyses are reported elsewhere[44]. In these analyzes, acute ischemic changes were classified according to a standard proforma[45]. Side of ischemic change was classified as right, left, or middle of the brain, and location was categorized according to three groups: middle cerebral artery (MCA) including any lesion in this vascular territory; anterior cerebral artery (ACA); and posterior cerebral artery (PCA) covering infratentorial locations such as brainstem, cerebellum, or both. For patients with multiple locations, they were included in each of the location categories. MCA was further classified as left or right side, and deep location. Mass effect was defined as the swelling compressing or displacing surrounding tissues, and graded according to a widely validated 7-point ordinal scale[46], with 0 indicating no swelling and 
scores from 1 to 6 indicating progressive increases in the size of swelling. The Alberta Stroke Program Early CT Score (ASPECTS)[47] measured the extent of ischemia in a range of 0-10: 10 representing no evidence of early ischemic change; 1 point being subtracted from 10 for any evidence of early ischemic change across defined regions of the MCA territory; and 0 indicating diffuse involvement throughout the MCA territory. An ASPECTS $>7$ was considered 'high' in these analyzes, according to other studies[48-50]. A large lesion was defined as the whole of the periphery, overall MCA territory, whole MCA and PCA territory, whole MCA and ACA territory, or all three vascular territories. The presence of tissue hypoattenuation, which indicates irreversible damage, was defined as either grey matter attenuation equal to normal white matter, or grey and white matter attenuation less than normal white matter[51]. Atrophy was present if there was any evidence of reduction in brain tissue volume, but grey and white matter volumes were not separately measured. White matter change included any periventricular white matter lucencies, hyperintensities, or both. Any presence of atrophy, white matter changes, or old infarct, was considered as background damage or 'frailty'.

\section{Statistical analysis}

Complete case analysis was used in developing various prediction models; patients with any missing values were excluded. The study population was randomly divided (2:1 ratio) into training (develop prediction models) and validation (validate predictive model performance) groups. Age (per 10-year increase), ethnicity, baseline NIHSS score, history of diabetes mellitus, pre-morbid mRS ( 0 or 1$)$ and proxy respondent, were selected a priori[52] to be forced into all models. Alteplase dose and onset to needle time did not show any significant association with HRQoL, hence these variables were not included in any model for these analyzes. Imaging features used to develop prediction models included acute ischaemic change (yes/no; right, left, middle; MCA, ACA and/or PCA, infratentorial locations; MCA 
territory left, right; MCA territory deep), mass effect, ASPECTS > 7, large infarct, hyperattenuated/abnormal vessel sign, tissue hypoattenuation, atrophy, white matter changes, old infarct, and any background damage.

Multivariable logistic regression was used to develop models using different combinations of variables according to different selection methods: in stepwise selection, the significance level for entry of a variable was set at $\mathrm{P}<0.2$, whereas the significance level for a variable staying was $\mathrm{P}<0.05$, and; for forward and backward selection, the significance level for variable entry was set as $\mathrm{P}<0.05$. Collinearity between variables were checked. Regression coefficients for models were estimated with maximum likelihood methods.

Discriminative ability of models was assessed using the concordance (C) statistic, which provides a range of $0.5-1.0[53]: 0.5$ represents a model no better than chance; $>0.7$ represents a good model; $>0.8$ represents a strong model; and 1 represents perfect fit for a model predicting an outcome. Receiver operating characteristic (ROC) curves and the HosmerLemeshow goodness of fit test $(\mathrm{P}<0.05$ indicates poor prediction or lack of fit) were used for comparisons of the performance of models in the development and validation datasets. Calibration plots provided visualisation of the level of agreement between observed and predicted probabilities: a calibration (fit) curve lying closely to the ideal line indicates that the model fits well with the data; a calibration curve away, or with systematic deviation, from the ideal line indicates that the model is not a good fit for the data. Bootstrapping of 1000 population samples simulated using random sampling with replacement was used to estimate the variability and precision of parameter estimates in the optimal models[54, 55]. Sampling distribution of regression coefficients were demonstrated in scatter plots, showing both variability of regression coefficients and correlated coefficients. Data are presented with odds ratios (OR) and 95\% confidence intervals (CI), and the significance level was set at 
$\mathrm{P}<0.05$ without adjustment for multiplicity. All analyses were undertaken using SAS enterprise (7.1).

\section{Results}

A total of 2526 ENCHANTED AIS patients (median age 67.5 years; $38.4 \%$ female; $61.7 \%$ Asian) had complete brain imaging data and 90-day EQ-5D utility scores available for analyzes (Figure 1). Included patients were less likely to be Asian, had milder neurological severity and had differences in their medical history and presumed AIS etiology when compared to excluded patients (Supplemental Table S1). Table 1 shows the characteristics of patients classified by good and poor HRQoL based on the utility score. Patients with poor HRQoL were significantly older, more proportion of female and non-Asian, had greater neurological impairment, more frequency of co-morbidities (hypertension, acute coronary syndrome, atrial fibrillation, diabetes mellitus and hypercholesterolaemia), pre-stroke disability (mRS score 1), current medication use (antihypertensive, aspirin, glucose-lowering, and statin agents), early ischemic change, right-sided lesions, in the MCA territory, with mass effect, poorer ASPECTS score $(<7)$, and were more likely to show infarction, hyperattenuated/abnormal vessel sign, tissue hypoattenuation, and background changes. There were no significant differences in the characteristics of patients in the training $(\mathrm{n}=1685)$ and validation $(\mathrm{n}=841)$ groups (Supplemental Table S2).

Eight different models were developed with significant imaging features selected and forced clinical factors (Supplemental Table S4): Models 1 and 4 included acute ischemic change in the right hemisphere; Model 2 included acute ischemic change in the middle and acute ischemic change in right hemisphere; Model 3 included acute ischemic change in the left hemisphere, mass effect, and tissue hypoattenuation; Model 5 included acute ischemic change in the left MCA territory, mass effect, and tissue hypoattenuation; Model 6 included 
acute ischemic change in the right MCA territory; Model 7 included acute ischemic change in the deep and right MCA territory, and; Model 8 included acute ischemic change in the left MCA territory and mass effect.

The performance of the various models was assessed in the validation dataset. Calibration plots show that the fit lines and 95\% CI for all the models, except Model 8, provide close coverage to the ideal line (Figure S1). However, the best calibration performance was for Model 7 which had a smoother fit line and less under-estimation of probability compared to the other models. Overall, though, there was consistency in the discrimination performance of models (C range 0.736 to 0.741 ; Figure S2), and all models except Model 8, had similar calibration and discrimination performance (Supplemental Figures S3 and S4). Table 2 shows the best fitted model Model 7, which included acute ischemic change in the right MCA territory (OR 1.69, 95\% 1.24-2.29) and in the deep MCA territory (OR 1.50, 95\%CI 1.032.19). From the 1000 simulated population samples using the bootstrapping technique, there was close and equal scattering of the parameter estimates in the training dataset, indicating good precision (Supplemental Table S5, Figure S5).

Table 3 shows results of multivariable logistic regression for predictors on various EQ-5D domains, with adjustment for baseline NIHSS scores and use of proxy respondents. Tissue hypoattenuation was significant for two domains: positive for poor mobility (OR 1.7, 95\% CI 1.08-2.68) and negative for pain/discomfort (OR 0.60, 95\% 0.39-0.93). Atrophy and white matter change also showed significant positive associations with poor mobility (OR 1.24, 95\% CI 1.02-1.51 and OR 1.52, 95\% CI 1.25-1.86, respectively). There were significant associations between each marker of background damage and poor self-care, and impaired usual activity: atrophy (OR 1.26, 95\% CI 1.01-1.56 and OR 1.33, 95\% CI 1.09-1.61, respectively); white matter change (OR 1.29, 95\% CI 1.04-1.60 and OR 1.26, 95\% CI 1.031.54, respectively) and old infarct (OR 1.24, 95\% CI 1.01-1.52 and OR 1.34, 95\% CI 1.11- 
1.62, respectively). However, no imaging features were associated with anxiety/depression. Complete Transparent Reporting of a multivariable prediction model for Individual Prognosis or Diagnosis (TRIPOD) checklist[56] is outlined in Supplemental Table S6.

\section{Discussion}

In these post-hoc analyses of a large multi-ethnic clinical population of thrombolysed AIS patients, brain imaging signs of right and deep MCA territory lesions stroke predicted poor overall HRQoL. While a variety of other imaging abnormalities - early ischemic change, MCA territory lesion, mass effect, large lesion, hyperattenuated/abnormal vessel sign, tissue hypoattenuation, and background changes - showed associations in univariate analyzes, they were not selected according to the pre-specified selection methods in multivariable analyzes with clinical factors for developing the optimal predictive model. Signs of brain frailty white matter change, atrophy and old infarcts - were each associated with most individual EQ-5D domains except anxiety/depression.

Associations between a wide range of brain imaging abnormalities, including subcortical and/or brainstem infarcts[31], severe subcortical grey matter hyperintensities[32], and infarct volume[30], and poor HRQoL have been reported in several small studies[30-32]. Studies involving several hundred of patients have shown that acute infarct volume predicts quality of life, except cognition[29], lobar cerebral microbleeds predict physical function and social function[33], deep white matter hyperintensities are related to overall HRQoL after lacunar AIS[34], and cerebral small vessel disease is associated with reduced quality of life[35]. However, all these studies used different questionnaires to measure HRQoL. Although the EQ-5D is not specific to stroke, it is one of the most popular, simple and broadly accepted approaches to measuring HRQoL in stroke trials. 
For patients with acute intracerebral hemorrhage, the findings of associations of hematomas located in the thalamus and infratentorial regions and poor HRQoL domains of mobility, selfcare and usual activity in the Intensive Blood Pressure Reduction in Acute Cerebral Haemorrhage Trial (INTERACT)[57], may reflect disruption of pyramidal tracts. The finding that right hemisphere AIS is associated with poor HRQoL might be explained by a range of deficits; parietal sensory symptoms including neglect, visuo-spatial deficits impairing navigation and dressing and language deficits including loss of comprehension of the emotional content. All of these features respond poorly to rehabilitation and if present, are likely to contribute to poor HRQoL[58,59]. Another possible explanation is reduced efficacy of thrombolysis in right hemispheric AIS[60, 61] and the deep MCA territories[62] where reduced reperfusion could translate into reduced HRQoL.

Strengths of our analyzes include the large sample, standardised measures and central blinded adjudication of brain imaging by trained assessors. We also undertook a full process of prediction model development and validation of imaging factors. Yet, our study was posthoc and limited to clinical trial data from thrombolyzed AIS patients of predominantly mildmoderate neurological severity, and with few patients who received endovascular therapy which evolved of the study period. Thus, selection bias was inevitable from these inclusion/exclusion criteria as well as from further exclusion of nearly one quarter of patients with missing scans or outcome, and from comparisons showing several significantly different variables across included and excluded patients. Another factor is that CT brain imaging significantly under-estimates early cerebral ischemia, and especially of the integrity of the pyramidal tracts, and also we did not include separate measurements of grey and white matter volumes. Finally, in any observational analysis, the strength and direction of association may have been influenced by residual confounding from clinical and/or imaging features. 
In conclusion, we found that right sided lesions and lesions including the deep volumes of the MCA location predicted poor overall HRQoL after AIS, whilst each background feature of brain frailty - atrophy, white matter change and old infarcts - is associated with adverse physical but not emotional HRQoL domains. These findings may assist in understanding the disconnect between physical function and quality of life as a whole to maximise recovery and wellbeing of AIS patients. 


\section{Author contributions}

$\mathrm{XC}$ and CD undertook analyses and wrote the draft; LS and CSA interpreted the data; all authors provided critical review and revisions, and approved submission of this article.

\section{Funding}

The ENCHANTED study was supported by grants from the National Health and Medical Research Council (NHMRC) of Australia (Project Grant numbers 1020462 and 1101113), the Stroke Association of the UK (TSA 2012/01 and 2015/01), Ministry of Health and the National Council for Scientific and Technological Development of Brazil (CNPQ: 467322/2014-7, 402388/2013-5), the Ministry for Health, Welfare and Family Affairs of the Republic of Korea (HI14C1985) (for the alteplase-dose arm), the National Institute for Health Research Clinical Research Network (NIHR CRN) for the conduct of the trial in England, UK and a research grant from Takeda to support the conduct of the blood pressure arm of the trial in China. During completion of this work CSA was supported by an NHMRC Senior Principal Research Fellowship, CD and XW were supported by a postdoctoral National Heart Foundation Fellowship, ZZ received overseas visiting funding from Ren Ji Hospital, School of Medicine, Shanghai Jiao Tong University (2016-2018) and a Scientia PhD Scholarship from the University of New South Wales, Sydney (2018-2022), MH was supported by a NHMRC Career Development Fellowship, and TGR was an NIHR Senior Investigator.

\section{Role of Sponsors}

The funding bodies had no role in the design and conduct of the study; collection, management, analyses, and interpretation of the data; and in preparation, review, or approval of the manuscript.

\section{Statement of Ethics}


This study complied with the guidelines for human studies. The research was conducted ethically in accordance with the World Medical Association Declaration of Helsinki.

\section{Conflicts of Interest Disclosures}

CSA reports receiving research grants and lecture fees from Takeda; JC reports research grants and lecture fees from Servier for the ADVANCE trial and post-trial follow-up; The other authors report no conflicts of interest.

\section{Acknowledgement}

We acknowledge the contribution of the large number of hospital investigators and coordinators, and central and regional project staff for the ENCHANTED study. We thank EuroQoL Group for providing translations and license for EQ-5D. Above all, we thank all patients, and their families and friends. 


\section{References}

1. Van der Worp HB, Kappelle $\amalg$. Complications of acute ischaemic stroke. Cerebrovasc Dis. 1998;8(2):124-32.

2. Hackett ML, Duncan JR, Anderson CS, Broad JB, Bonita R. Health-related quality of life among long-term survivors of stroke : results from the Auckland Stroke Study, 19911992. Stroke; a journal of cerebral circulation. 2000;31(2):440-7.

3. Dhamoon MS, Moon YP, Paik MC, Boden-Albala B, Rundek T, Sacco RL, et al. Quality of life declines after first ischemic stroke. The Northern Manhattan Study. Neurology. 2010;75(4):328-34.

4. Sturm JW, Donnan GA, Dewey HM, Macdonell RA, Gilligan AK, Thrift AG.

Determinants of handicap after stroke: the North East Melbourne Stroke Incidence Study (NEMESIS). Stroke. 2004;35(3):715-20.

5. Wang R, Langhammer B. Predictors of quality of life for chronic stroke survivors in relation to cultural differences: a literature review. Scandinavian Journal of Caring Sciences. 2018;32(2):502-14.

6. Kauhanen ML, Korpelainen JT, Hiltunen P, Nieminen P, Sotaniemi KA, Myllyla VV. Domains and determinants of quality of life after stroke caused by brain infarction. Arch Phys Med Rehabil. 2000;81(12):1541-6.

7. Reeves SL, Brown DL, Baek J, Wing JJ, Morgenstern LB, Lisabeth LD. Ethnic Differences in Poststroke Quality of Life in the Brain Attack Surveillance in Corpus Christi (BASIC) Project. Stroke. 2015;46(10):2896-901.

8. Kim P, Warren S, Madill H, Hadley M. Quality of life of stroke survivors. Qual Life Res. 1999;8(4):293-301.

9. Clarke $P$, Marshall V, Black SE, Colantonio A. Well-being after stroke in Canadian seniors: findings from the Canadian Study of Health and Aging. Stroke. 2002;33(4):1016-21.

10. Lai SM, Studenski S, Duncan PW, Perera S. Persisting consequences of stroke measured by the Stroke Impact Scale. Stroke. 2002;33(7):1840-4.

11. Kostadinova E, Anita A. Influence of depression on the quality of life after stroke. European Psychiatry. 2017;41(sS):S475-S.

12. Wulsin L, Alwell K, Moomaw CJ, Lindsell CJ, Kleindorfer DO, Woo D, et al. Comparison of two depression measures for predicting stroke outcomes. Journal of Psychosomatic Research. 2012;72(3):175-9.

13. Milone G, Balucani C, Hong S, Stefanov D, Levine S. Interplay of depression and fatigue on quality of life after stroke. Journal of the Neurological Sciences.

2015;357(S1):e392-e.

14. Morris JH, van Wijck F, Joice S, Donaghy M. Predicting health related quality of life 6 months after stroke: the role of anxiety and upper limb dysfunction. Disabil Rehabil.

2013;35(4):291-9.

15. Jonkman EJ, de Weerd AW, Vrijens NL. Quality of life after a first ischemic stroke. Long-term developments and correlations with changes in neurological deficit, mood and cognitive impairment. Acta neurologica Scandinavica. 1998;98(3):169-75.

16. Schmid AA, Van Puymbroeck M, Altenburger P, Miller K, Combs S. Balance Impairment Is Associated with Decreased Quality of Life in People with Chronic Stroke. Archives of Physical Medicine and Rehabilitation. 2012;93(10):e12-e. 
17. Akpalu A, Calys-Tagoe B, RN. K-N. The Effect of Cognitive Impairment on the HealthRelated Quality of Life Among Stroke Survivors at a Major Referral Hospital in Ghana. West African Journal of Medicine. 2018(35(3)):199-203.

18. Pickard AS, Johnson JA, Feeny DH, Shuaib A, Carriere KC, Nasser AM. Agreement between patient and proxy assessments of health-related quality of life after stroke using the EQ-5D and Health Utilities Index. Stroke. 2004;35(2):607-12.

19. Sangha RS, Caprio FZ, Askew R, Corado C, Bernstein R, Curran Y, et al. Quality of life in patients with TIA and minor ischemic stroke. Neurology. 2015;85(22):1957-63.

20. Delcourt C, Zheng D, Chen X, Hackett M, Arima H, Hata J, et al. Associations with health-related quality of life after intracerebral haemorrhage: pooled analysis of INTERACT studies. J Neurol Neurosurg Psychiatry. 2017;88(1):70-5.

21. Kwok T, Lo RS, Wong E, Wai-Kwong T, Mok V, Kai-Sing W. Quality of life of stroke survivors: a 1-year follow-up study. Arch Phys Med Rehabil. 2006;87(9):1177-82; quiz 287.

22. Brajkovic L, Godan A, Godan L. Quality of life after stroke in old age: comparison of persons living in nursing home and those living in their own home.(PUBLIC HEALTH)(Report). Croatian Medical Journal. 2009;50(2):182.

23. Mackenzie AE, Chang AM. Predictors of quality of life following stroke. Disability and Rehabilitation. 2002;24(5):259-65.

24. Ellis C, Grubaugh AL, Egede LE. Factors associated with SF-12 physical and mental health quality of life scores in adults with stroke. J Stroke Cerebrovasc Dis. 2013;22(4):309-

17.

25. Tse T, Binte Yusoff SZ, Churilov L, Ma H, Davis S, Donnan GA, et al. Increased work and social engagement is associated with increased stroke specific quality of life in stroke survivors at 3 months and 12 months post-stroke: a longitudinal study of an Australian stroke cohort. Topics in Stroke Rehabilitation. 2017;24(6):405-14.

26. Tariah HA, Hersch G, Ostwald SK. Factors Associated with Quality of Life: Perspectives of Stroke Survivors. Physical \& Occupational Therapy In Geriatrics. 2006;25(2):33-50.

27. Darlington A-S, Ribbers G, Passchier J. Coping Strategies as Determinants of Quality of Life in Stroke Patients: A Longitudinal Study. Cerebrovascular Diseases. 2007;23(5-6):4017.

28. Clarke P, Black SE. Quality of Life Following Stroke: Negotiating Disability, Identity, and Resources. The Journal of Applied Gerontology. 2005;24(4):319-36.

29. Lin C, Lee J, Chatterjee N, Corado C, Carroll T, Naidech A, et al. Predicting DomainSpecific Health-Related Quality of Life Using Acute Infarct Volume. Stroke. 2017;48(7):192531.

30. Schiemanck SK, Post MW, Kwakkel G, Witkamp TD, Kappelle L, Prevo AJ. Ischemic lesion volume correlates with long-term functional outcome and quality of life of middle cerebral artery stroke survivors. Restor Neurol Neurosci. 2005;23(3-4):257-63.

31. Lin C, Sangha R, Lee J, Corado C, Jalasutram A, Chatterjee N, et al. Infarct location is associated with quality of life after mild ischemic stroke. Int J Stroke. 2018;13(8):824-31.

32. Moon YS, Kim SJ, Kim HC, Won MH, Kim DH. Correlates of quality of life after stroke. J Neurol Sci. 2004;224(1-2):37-41.

33. Tang WK, Chen YK, Lu J, Ahuja AT, Chu WC, Mok VC, et al. Cerebral microbleeds and quality of life in acute ischemic stroke. Neurol Sci. 2011;32(3):449-54.

34. Tang WK, Liang HJ, Chen YK, Ahuja AT, Chu WC, Mok VC, et al. White matter hyperintensities and quality of life in acute lacunar stroke. Neurol Sci. 2013;34(8):1347-53. 
35. Liang $\mathrm{Y}$, Chen YK, Deng M, Mok VCT, Wang DF, Ungvari GS, et al. Association of Cerebral Small Vessel Disease Burden and Health-Related Quality of Life after Acute Ischemic Stroke. Front Aging Neurosci. 2017;9:372.

36. Anderson CS, Huang $\mathrm{Y}$, Lindley RI, Chen $\mathrm{X}$, Arima $\mathrm{H}$, Chen $\mathrm{G}$, et al. Intensive blood pressure reduction with intravenous thrombolysis therapy for acute ischaemic stroke (ENCHANTED): an international, randomised, open-label, blinded-endpoint, phase 3 trial. The Lancet. 2019;393(10174):877-88.

37. Anderson CS, Robinson T, Lindley RI, Arima H, Lavados PM, Lee TH, et al. Low-Dose versus Standard-Dose Intravenous Alteplase in Acute Ischemic Stroke. N Engl J Med. 2016;374(24):2313-23.

38. Anderson CS, Woodward M, Arima H, Chen X, Lindley RI, Wang X, et al. Statistical analysis plan for evaluating low- vs. standard-dose alteplase in the ENhanced Control of Hypertension and Thrombolysis strokE stuDy (ENCHANTED). Int J Stroke. 2015;10(8):1313-5. 39. Anderson CS, Woodward M, Arima H, Chen X, Lindley RI, Wang X, et al. Statistical analysis plan for evaluating different intensities of blood pressure control in the ENhanced Control of Hypertension And Thrombolysis strokE stuDy. Int J Stroke.

2018:1747493018806170.

40. Huang Y, Sharma VK, Robinson T, Lindley RI, Chen X, Kim JS, et al. Rationale, design, and progress of the ENhanced Control of Hypertension ANd Thrombolysis strokE stuDy (ENCHANTED) trial: An international multicenter $2 \times 2$ quasi-factorial randomized controlled trial of low- vs. standard-dose rt-PA and early intensive vs. guideline-recommended blood pressure lowering in patients with acute ischaemic stroke eligible for thrombolysis treatment. Int J Stroke. 2015;10(5):778-88.

41. EuroQol G. EuroQol--a new facility for the measurement of health-related quality of life. Health Policy. 1990;16(3):199-208.

42. Dolan P, Gudex C, Kind P, Williams A. A Social Tariff for EuroQoL: Results from a UK General Population Survey. 1995.

43. Dolan P. Modeling valuations for EuroQol health states. Medical Care. 1997;35(11 (Nov., 1997)):1095-108.

44. Delcourt C, Wang X, Zhou Z, Wardlaw J, Mair G, Robinson T, et al. Brain imaging abnormalities and outcome after acute ischaemic stroke: the ENCHANTED trial. Submitted to Journal of Neurology, Neurosurgery \& Psychiatry. 2020.

45. Wardlaw JM, Sellar R. A simple practical classification of cerebral infarcts on CT and its interobserver reliability. AJNR Am J Neuroradiol. 1994;15(10):1933-9.

46. Kharitonova T, Ahmed N, Thoren M, Wardlaw JM, von Kummer R, Glahn J, et al. Hyperdense middle cerebral artery sign on admission CT scan--prognostic significance for ischaemic stroke patients treated with intravenous thrombolysis in the safe implementation of thrombolysis in Stroke International Stroke Thrombolysis Register. Cerebrovasc Dis. 2009;27(1):51-9.

47. Barber PA, Demchuk AM, Zhang J, Buchan AM. Validity and reliability of a quantitative computed tomography score in predicting outcome of hyperacute stroke before thrombolytic therapy. ASPECTS Study Group. Alberta Stroke Programme Early CT Score. Lancet. 2000;355(9216):1670-4.

48. Demchuk AM, Hill MD, Barber PA, Silver B, Patel SC, Levine SR, et al. Importance of early ischemic computed tomography changes using ASPECTS in NINDS rtPA Stroke Study. Stroke. 2005;36(10):2110-5. 
49. Hill MD, Demchuk AM, Goyal M, Jovin TG, Foster LD, Tomsick TA, et al. Alberta Stroke Program early computed tomography score to select patients for endovascular treatment: Interventional Management of Stroke (IMS)-III Trial. Stroke. 2014;45(2):444-9. 50. Group TI-c. Association between brain imaging signs, early and late outcomes, and response to intravenous alteplase after acute ischaemic stroke in the third International Stroke Trial (IST-3): secondary analysis of a randomised controlled trial. The Lancet Neurology. 2015;14(5):485-96.

51. Wardlaw JM, Mielke O. Early signs of brain infarction at CT: observer reliability and outcome after thrombolytic treatment--systematic review. Radiology. 2005;235(2):444-53. 52. Chen X, Wang X, Delcourt C, Li J, Arima H, Hackett $M$, et al. Ethnicity and Other Determinants of Quality of Functional Outcome in Acute Ischemic Stroke: The ENCHANTED Trial. Stroke. 2020;51(2):588-93.

53. Tripepi G, Jager KJ, Dekker FW, Zoccali C. Statistical methods for the assessment of prognostic biomarkers (Part I): discrimination. Nephrol Dial Transplant. 2010;25(5):1399401.

54. Cassell DL. BootstrapMania!: Re-Sampling the SAS Way. Statistics and Data Analysis. 2010;SAS Global Forum 2010:Paper 268-2010.

55. Laberge Y. Advising on Research Methods: A Consultant's Companion. Journal of Applied Statistics. 2011;38(12):2991-.

56. Collins GS, Reitsma JB, Altman DG, Moons KG, Group T. Transparent reporting of a multivariable prediction model for individual prognosis or diagnosis (TRIPOD): the TRIPOD statement. The TRIPOD Group. Circulation. 2015;131(2):211-9.

57. Delcourt C, Sato S, Zhang S, Sandset E, Zheng D, Chen X, et al. Intracerebral hemorrhage location and outcome among INTERACT2 participants. Neurology.

2017;88(15):1408-14.

58. Azouvi $P$, Jacquin-Courtois $S$, Luaute J. Rehabilitation of unilateral neglect: Evidencebased medicine. Ann Phys Rehabil Med. 2017;60(3):191-7.

59. Müri R, Cazzoli D, Nef T, Hopfner S, Nyffeler T. Non-Invasive Brain Stimulation in Neglect Rehabilitation: An Update. Frontiers in Human Neuroscience. 2013;7(MAY).

60. Foerch C, Misselwitz B, Sitzer M, Berger K, Steinmetz H, Neumann-Haefelin T, et al. Difference in recognition of right and left hemispheric stroke. Lancet. 2005;366(9483):392-3. 61. Di Legge S, Fang J, Saposnik G, Hachinski V. The impact of lesion side on acute stroke treatment. Neurology. 2005;65(1):81-6.

62. Rosso C, Colliot O, Valabregue R, Crozier S, Dormont D, Lehericy S, et al. Tissue at risk in the deep middle cerebral artery territory is critical to stroke outcome. Neuroradiology. 2011;53(10):763-71. 


\section{Figures legend}

Figure 1. Flowchart of patients included for analyzes 
Table 1 Patient characteristics by level of health-related quality of life (HRQoL)

\begin{tabular}{|c|c|c|c|}
\hline \multirow[b]{2}{*}{ Variables } & \multicolumn{2}{|c|}{ HRQoL* } & \multirow[b]{2}{*}{$P$ value } \\
\hline & $\begin{array}{c}\text { Good } \\
(n=1717)\end{array}$ & $\begin{array}{c}\text { Poor } \\
(n=809)\end{array}$ & \\
\hline Age, years & $65.8(56.8-74.4)$ & $70.4(61.6-78.3)$ & $<0.0001$ \\
\hline Male & $1112 / 1717(64.8)$ & $444 / 809(54.9)$ & $<0.0001$ \\
\hline Asian & $1108 / 1717(64.5)$ & $450 / 809(55.6)$ & $<0.0001$ \\
\hline \multicolumn{4}{|l|}{ Clinical measures } \\
\hline SBP & $151(135-164)$ & $152(137-168)$ & 0.020 \\
\hline DBP & $84(76-93)$ & $84(76-93)$ & 0.964 \\
\hline HR & $78(69-86)$ & $78(68-88)$ & 0.732 \\
\hline NIHSS score & $7(4-11)$ & $11(6-16)$ & $<0.0001$ \\
\hline GCS score & $15(14-15)$ & $15(13-15)$ & $<0.0001$ \\
\hline \multicolumn{4}{|l|}{ Medical history } \\
\hline Hypertension & $1053 / 1716(61.4)$ & $535 / 809(66.1)$ & 0.021 \\
\hline Previous stroke & $300 / 1717(17.5)$ & $136 / 809(16.8)$ & 0.682 \\
\hline Coronary artery disease & $220 / 1716(12.8)$ & $131 / 809(16.2)$ & 0.022 \\
\hline Atrial fibrillation & $269 / 1715(15.7)$ & $193 / 809(23.9)$ & $<0.0001$ \\
\hline Diabetes & $300 / 1716(17.5)$ & $184 / 809(22.7)$ & 0.002 \\
\hline Hypercholesterolaemia & $270 / 1716(15.7)$ & $182 / 809(22.5)$ & $<0.0001$ \\
\hline Current smoker & $442 / 1715(25.8)$ & $182 / 808(22.5)$ & 0.078 \\
\hline Pre-stroke modified Rankin scale score 1 & $251 / 1716(14.6)$ & $219 / 808(27.1)$ & $<0.0001$ \\
\hline \multicolumn{4}{|l|}{ Medications } \\
\hline Antihypertensive agents & $763 / 1716(44.5)$ & $419 / 809(51.8)$ & 0.001 \\
\hline Warfarin anticoagulation & $35 / 1715(2.0)$ & $24 / 807(3.0)$ & 0.148 \\
\hline Aspirin or other antiplatelet agent & $365 / 1715(21.3)$ & $231 / 807(28.6)$ & $<0.0001$ \\
\hline Glucose lowering agent(s) & $195 / 1715(11.4)$ & $123 / 807(15.2)$ & 0.006 \\
\hline Statin or other lipid lowering agent & $304 / 1714(17.7)$ & $196 / 807(24.3)$ & 0.0001 \\
\hline \multicolumn{4}{|l|}{ Final diagnosist } \\
\hline Stroke mimic & $65 / 1714(3.8)$ & $16 / 808(2.0)$ & 0.016 \\
\hline \multicolumn{4}{|l|}{ Stroke cause } \\
\hline Large artery occlusive etiology & $578 / 1714(33.7)$ & $328 / 808(40.6)$ & 0.001 \\
\hline Small vessel disease & $471 / 1714(27.5)$ & $116 / 808(14.4)$ & $<0.0001$ \\
\hline Cardioembolism & $302 / 1714(17.6)$ & $200 / 808(24.8)$ & $<0.0001$ \\
\hline Other or uncertain etiology & $298 / 1714(17.4)$ & $148 / 808(18.3)$ & 0.568 \\
\hline Randomized to low-dose alteplase & $861 / 1717(50.2)$ & $420 / 809(51.9)$ & 0.406 \\
\hline Proxy respondent & $566 / 1717(33.0)$ & $443 / 809(54.8)$ & $<0.0001$ \\
\hline \multicolumn{4}{|l|}{ Brain imaging features } \\
\hline Acute ischaemic change & $508 / 1717(29.6)$ & $313 / 809(38.7)$ & $<0.0001$ \\
\hline \multicolumn{4}{|l|}{ Side of ischaemic change } \\
\hline Right & 209/1717 (12.2) & $154 / 809(19.0)$ & $<0.0001$ \\
\hline Left & $297 / 1717(17.3)$ & $159 / 809(19.7)$ & 0.151 \\
\hline Middle & $4 / 1717(0.2)$ & $2 / 809(0.3)$ & 0.945 \\
\hline \multicolumn{4}{|l|}{ Classification of ischemic change } \\
\hline Grey/white matter cortex & $304 / 1127(27.0)$ & $185 / 507(36.5)$ & 0.0001 \\
\hline Basal ganglia outline & $243 / 1127(21.6)$ & $147 / 507(29.0)$ & 0.001 \\
\hline Hypodensity & $341 / 1127(30.3)$ & $197 / 507(38.9)$ & 0.001 \\
\hline \multicolumn{4}{|l|}{ Location of ischemic lesion } \\
\hline MCA & $488 / 1714(28.5)$ & $300 / 809(37.1)$ & $<0.0001$ \\
\hline Deep MCA & $157 / 1714(9.2)$ & $94 / 809(11.6)$ & 0.054 \\
\hline
\end{tabular}


Left MCA

Right MCA

ACA and/or PCA

Infratentorial (cerebellum or brainstem)

Mass effect

ASPECTS

ASPECTS $>7$

Large infarct

Hyperattenuated/abnormal vessel sign

Tissue hypoattenuation

Any background damage

Atrophy

Old infarct

White matter changes

Anterior white matter

Posterior white matter

\begin{tabular}{ccc}
$289 / 1717(16.8)$ & $153 / 809(18.9)$ & 0.217 \\
$200 / 1717(11.7)$ & $147 / 809(18.2)$ & $<0.0001$ \\
$15 / 1714(0.9)$ & $15 / 809(1.9)$ & 0.034 \\
$10 / 1714(0.6)$ & $5 / 809(0.6)$ & 0.916 \\
$512 / 1717(29.8)$ & $318 / 809(39.3)$ & $<0.0001$ \\
$8(5-9)$ & $8(5-9)$ & 0.740 \\
$1497 / 1717(87.2)$ & $670 / 809(82.8)$ & 0.003 \\
$46 / 1707(2.7)$ & $43 / 803(5.4)$ & 0.001 \\
$221 / 1714(12.9)$ & $158 / 809(19.5)$ & $<0.0001$ \\
$443 / 1712(25.9)$ & $264 / 809(32.6)$ & 0.0004 \\
$1210 / 1717(70.5)$ & $655 / 809(81.0)$ & $<0.0001$ \\
$995 / 1717(58.0)$ & $553 / 809(68.4)$ & $<0.0001$ \\
$563 / 1714(32.9)$ & $324 / 809(40.1)$ & 0.0004 \\
$492 / 1717(28.7)$ & $308 / 809(38.1)$ & $<0.0001$ \\
$414 / 1673(24.8)$ & $250 / 783(31.9)$ & 0.0002 \\
$329 / 1674(19.6)$ & $216 / 783(27.6)$ & $<0.0001$ \\
\hline
\end{tabular}

Data are $\mathrm{n}(\%)$, or median (IQR). P values are based on Chi-square or Mann-Whitney tests.

ACA denotes anterior cerebral artery, ASPECTS Alberta Stroke Program Early CT Score, DBP diastolic blood pressure, GCS Glasgow coma scale, HR heart rate, HRQoL health related quality of life, MCA middle cerebral artery, SBP systolic blood pressure, NIHSS National Institute of Health Stroke Scale, mRS modified Rankin scale, PCA posterior cerebral artery

*Level of HRQoL defined as good or poor according to $>0.7$ or $\leq 0.7$ mean utility score on the 5-dimension European Quality of Life scale (EQ-5D) 
Table 2 Associations between imaging features and poor HRQoL in training dataset

\begin{tabular}{|c|c|c|c|c|c|c|}
\hline \multirow[b]{2}{*}{ Variables } & \multicolumn{2}{|c|}{ EQ-5D utility score* } & \multicolumn{2}{|c|}{ Univariable } & \multicolumn{2}{|c|}{ Model 7† } \\
\hline & $>0.7(n=1145)$ & $\leq 0.7(n=540)$ & OR $(95 \% C I)$ & P value & OR (95\%CI) & P value \\
\hline Acute ischemic change & $327 / 1145(28.6)$ & $213 / 540(39.4)$ & $1.63(1.31-2.02)$ & $<0.0001$ & & \\
\hline \multicolumn{7}{|l|}{ Side of ischemic change } \\
\hline Right & $132 / 1145(11.5)$ & $103 / 540(19.1)$ & $1.81(1.37-2.40)$ & $<0.0001$ & & \\
\hline Left & $198 / 1145(17.3)$ & $110 / 540(20.4)$ & $1.22(0.94-1.59)$ & 0.128 & & \\
\hline Middle & $1 / 1145(0.1)$ & $2 / 540(0.4)$ & $4.25(0.39-47.0)$ & 0.238 & & \\
\hline \multicolumn{7}{|l|}{ Location of ischemic lesion } \\
\hline $\mathrm{MCA}$ & $317 / 1143(27.7)$ & 204/540 (37.8) & $1.58(1.27-1.97)$ & $<0.0001$ & & \\
\hline Left & $193 / 1143$ (16.9) & $106 / 540(19.6)$ & $1.21(0.93-1.57)$ & 0.165 & & \\
\hline Right & $126 / 1143(11.0)$ & $98 / 540(18.2)$ & $1.79(1.35-2.39)$ & $<0.0001$ & $1.46(1.05-2.02)$ & 0.02 \\
\hline Deep MCA & $92 / 1143(8.1)$ & $70 / 540(13.0)$ & $1.70(1.23-2.37)$ & 0.002 & $1.50(1.03-2.19)$ & 0.03 \\
\hline $\mathrm{ACA}$ and/or PCA & 9/1143 (0.8) & 10/540 (1.9) & $2.38(0.96-5.88)$ & 0.061 & & \\
\hline Infratentorial & $4 / 1143(0.4)$ & $4 / 540(0.7)$ & $2.13(0.53-8.53)$ & 0.288 & & \\
\hline Mass effect & $330 / 1145(28.8)$ & $217 / 540(40.2)$ & $1.66(1.34-2.06)$ & $<0.0001$ & & \\
\hline ASPECTS & $7(5-9)$ & $8(5-9)$ & $1.04(0.96-1.12)$ & 0.368 & & \\
\hline ASPECTS >7 & $991 / 1145(86.6)$ & $448 / 540(83.0)$ & $0.76(0.57-1.00)$ & 0.052 & & \\
\hline Large infarct & $32 / 1140(2.8)$ & 25/536 (4.7) & $1.69(0.99-2.89)$ & 0.053 & & \\
\hline Hyperattenuated/abnormal vessel sign & $160 / 1143(14.0)$ & $102 / 540(18.9)$ & $1.43(1.09-1.88)$ & 0.010 & & \\
\hline Tissue hypoattenuation & $290 / 1142(25.4)$ & $178 / 540(33.0)$ & $1.45(1.16-1.81)$ & 0.001 & & \\
\hline Markers of background damage & $811 / 1145(70.8)$ & $437 / 540(80.9 S)$ & $1.75(1.36-2.24)$ & $<0.0001$ & & \\
\hline Atrophy & $677 / 1145(59.1)$ & $374 / 540(69.3)$ & $1.56(1.25-1.94)$ & $<0.0001$ & & \\
\hline White matter changes & $318 / 1145(27.8)$ & $195 / 540(36.1)$ & $1.47(1.18-1.83)$ & 0.0005 & & \\
\hline Old infarct & $363 / 1143(31.8)$ & $216 / 540(40.0)$ & $1.43(1.161 .77)$ & 0.0009 & & \\
\hline
\end{tabular}

ACA denotes anterior cerebral artery, ASPECTS Alberta Stroke Program Early CT Score, CI confidence interval, EQ-5D 5-dimension European Quality of life scale HRQoL health related quality of life, MCA middle cerebral artery, OR odds ratio, PCA posterior cerebral artery

*EQ-5D mean utility scores divided by $>0.7$ or $\leq 0.7$ mean value

$\dagger$ Model 7 consists of log odds of poor HRQoL $=-1.658+0.099 *$ age $/ 10-0.255^{*}$ Asian $+0.094 *$ National Institutes of Health stroke scale (NIHSS) score $+0.186 *$ diabetes mellitus $+0.256 *$ modified Rankin scale $(\mathrm{mRS})$ score $+0.355 *$ proxy respondent $+0.188 *$ right $\mathrm{MCA}+0.204 *$ deep $\mathrm{MCA}$ 
Table 3. Multivariable logistic regression analyses showing OR $(95 \% \mathrm{CI})$ for associations of brain imaging features and dimensions (some/moderate or severe problems vs. no problem; 2 and 3 vs. 1) on the EQ-5D scale

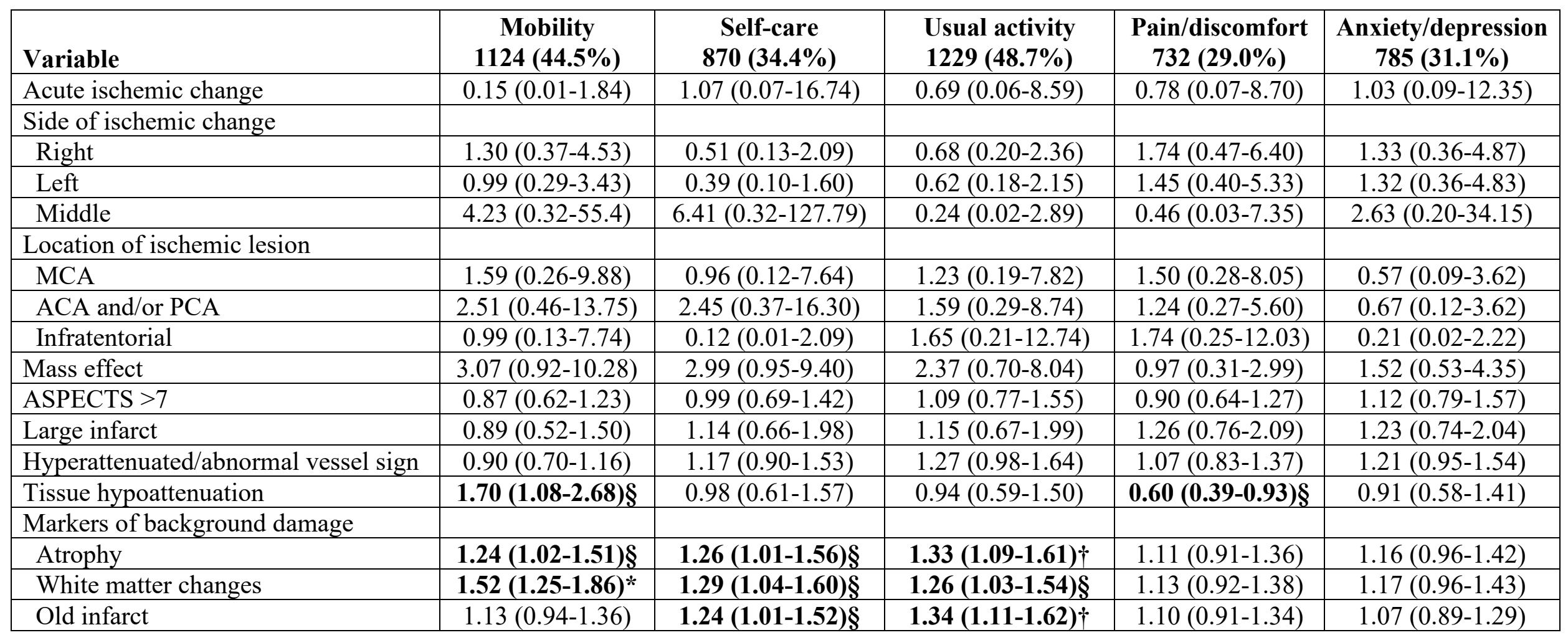

ACA denotes anterior cerebral artery, ASPECTS Alberta stroke program early CT score, CI confidence interval, MCA denotes middle cerebral artery, OR odds ratio, PCA posterior cerebral artery,

Models adjusted for baseline National Institutes of Health stroke scale (NIHSS) score and proxy respondent.

$* \mathrm{P}<0.0001$

$\dagger \mathrm{P}<0.01$

$\S \mathrm{P}<0.05$ 
Figure 1 Flowchart of patients included for analyzes

3310 patients were randomized in the alteplase-dose arm of the ENCHANTED trial

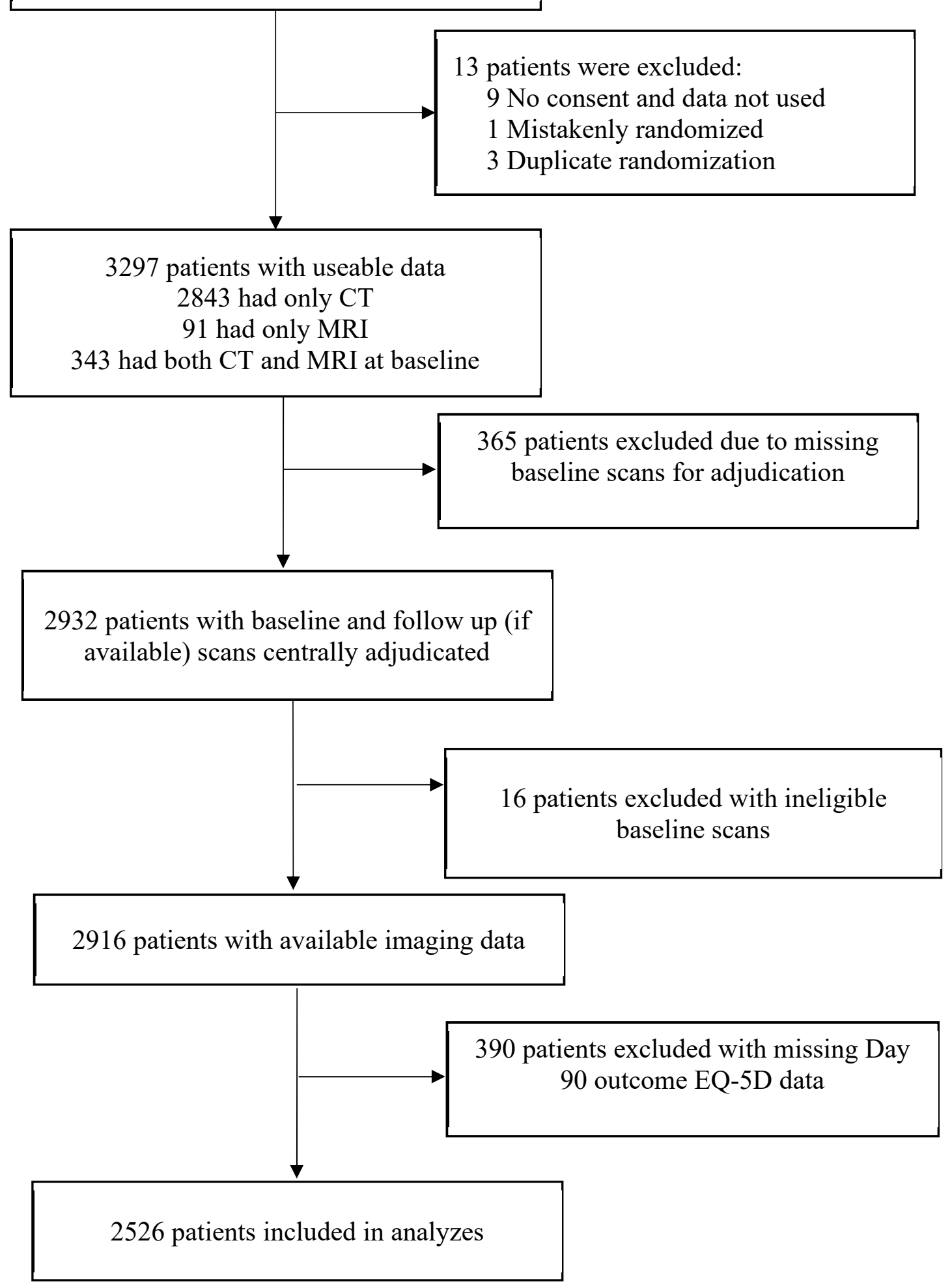

